\title{
CONSTRUCTION OF GREEN TIDE MONITORING SYSTEM AND RESEARCH ON ITS KEY TECHNIQUES
}

\author{
Xing Baiyang ${ }^{1, *}$, Li Jiagui ${ }^{1}$, Zhu Hui ${ }^{1}$, Wei Pan $^{1}$, Zhao Yingjie
}

1. College of Geomatics, Shandong University of Science and Technology, Qingdao, 266510, China;

KEY WORDS: green tide, GIS, remote sensing, integration, spatial distribution extraction

\begin{abstract}
:
As a kind of marine natural disaster, Green Tide has been appearing every year along the Qingdao Coast, bringing great loss to this region, since the large-scale bloom in 2008. Therefore, it is of great value to obtain the real time dynamic information about green tide distribution. In this study, methods of optical remote sensing and microwave remote sensing are employed in Green Tide Monitoring Research. A specific remote sensing data processing flow and a green tide information extraction algorithm are designed, according to the optical and microwave data of different characteristics. In the aspect of green tide spatial distribution information extraction, an automatic extraction algorithm of green tide distribution boundaries is designed based on the principle of mathematical morphology dilation/erosion. And key issues in information extraction, including the division of green tide regions, the obtaining of basic distributions, the limitation of distribution boundary, and the elimination of islands, have been solved. The automatic generation of green tide distribution boundaries from the results of remote sensing information extraction is realized. Finally, a green tide monitoring system is built based on IDL/GIS secondary development in the integrated environment of RS and GIS, achieving the integration of RS monitoring and information extraction.
\end{abstract}

\section{INTRODUCTION}

Green Tide is a kind of marine ecological anomaly occurring generally in coastal areas around the world. It is an abnormal ecological phenomenon caused by large scale proliferation of Ulva and Enteromorpha and other kinds of algae (Hiraoka et al., 2004; Nelson et al., 2003). In recent years, the Yellow Sea, especially the coastal areas from Jiangsu Province to Yantai, Shandong Province, Chi-na, have been repeatedly disturbed by large-scale green tides (Hu et al., 2008; Liu et al., 2016). The ecological environment there is greatly damaged and the seafood industry suffers great economical losses when green tide breaks out (Liu et

${ }^{*}$ Corresponding author.

E-mail address: 1778278423@qq.com (XING BaiYang) al., 2009). Meanwhile, it will cause further pollution to marine environment after the green tide begins to decay(Quillien et al., 2015; Jeffrey et al., 1993). Therefore, it has become a significant research direction for the maritime sectors to figure out how to carry out green tide monitoring and early warning.

Remote sensing image has natural advantages in regional environment monitoring for its characteristics of large scale, multi temporality\& high efficiency. With the vigorous development of satellite remote sensing techniques, it has become a feasible and ideal way to conduct large-scale marine monitoring using remote sensing data (Wang et al., 1998). While GIS has a strong ability to manage geodata and to conduct spatial 
analysis and visualization with geodata, which makes it effective in managing huge amount of multi-period, multi-source remote sensing data in different formats and extracting the distribution information and its contour of green tide through spatial analysis. However, the present-used green tide monitoring method that combines remote sensing and GIS is complicated. Meanwhile the level of automation is low and the method relies a lot on professional platform and manual operation, resulting in low work efficiency. There lacks research on an automatic, integrated system which is needed in green tide monitoring business.

Therefore, in this study, a green tide monitoring system where remote sensing data and algorithms are integrated into GIS environment, is designed and developed to greatly improve the efficiency in the procedure from data processing to product making.

\section{FRAMEWORK OF THE SYSTEM}

The green tide monitoring system utilizes ENVI/ IDL, which is professional in image processing, to create the remote sensing processing modules (Kong et al., 2013; Wang et al., 2011; Wang, 2011).The system sets ArcGIS10.1 as its GIS environment and realizes the extraction of green tide distribution information and monitoring results display based on ArcEngine10.1 and C\#.

Two kinds of optical remote sensing data (MODIS, HJ-1 CCD) and three kinds of microwave remote sensing data (COSMO, RADARSAT, Terra SAR-X) are utilized comprehensively in order to realize largescale, high-precise, all-weather green tide monitoring in this study. And remote sensing data preprocessing algorithms for different types of data and green tide coverage information extraction algorithm are designed and sealed as independent modules. These modules are called to obtain the bi-value images that contain green tide coverage information under the GIS environment (Wang et al., 2014). Then Euclidean Distance Analysis and Closing Mathematical Morphology—Dilation/Erosion are combined to extract the spatial distribution information from the green tide coverage bi-value images. At last, specific products are made with the support of GIS for its outstanding ability of data display and map-making (Dekker et al., 1993; Wang, 2010). Basic process is shown in Fig.1.

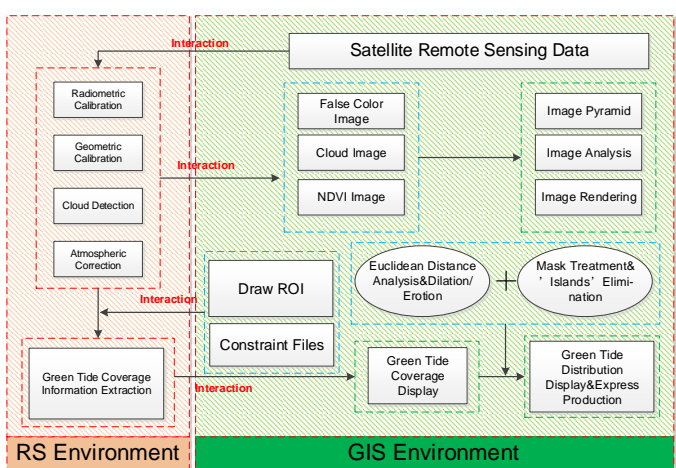

Figure.1 Flow Chart of Green Tide Monitoring System

These two main questions are focused on in this paper:

(1) Integration of GIS and remote sensing

In the traditional methods to extract distribution information from multi-source remote sensing data, a series of pre-processing operations are needed first in professional remote sensing software, then algorithms are designed for coverage information extraction. After that the results are transferred into GIS environment to conduct spatial analysis with to obtain the green tide distribution information and display it.

(2) Green tide distribution contour extraction

The contour of the green tide is needed because there is no accurate green tide distribution contour information in the bi-value images extracted by green tide coverage information extracting algorithm and area of distribution cannot be calculated through this kind of images. Euclidean distance analysis and mathematical morphology closed operation(dilation/erosion) are combined to obtain accurate enveloping polygons of green tide coverage points.

\section{INTEGRATION OF GIS AND REMOTE SENSING}

The basic framework of green tide monitoring system is to extract green tide coverage information by remote sensing information extracting module and to achieve 
the data organization \& display and product making based on GIS. Remote sensing processing modules are embedded in GIS environment and the information interaction between remote sensing processing and GIS analysis is achieved. In order to realize better fusion of the two environments and data interaction, the following are done:

(1) Packaging and calling of remote sensing image processing modules:

As the loose integration based on executable program(EXE) has the advantages of cross platform, flexibility, convenience and portability, image processing module sealed as EXE is more conducive to integration with GIS environment and data interaction. Loose integration way is adopted to carry on secondary development and remote sensing image processing modules are sealed to obtain the corresponding EXEs. Information about these image processing modules is summarized in Table 1. In the system, the remote sensing image processing module EXEs are invoked by the GIS platform through configuration files.

\begin{tabular}{|c|c|c|c|c|}
\hline Processing Modules & $\begin{array}{l}\text { Input Data Types } \\
\text { (Format) }\end{array}$ & $\begin{array}{l}\text { Reserved } \\
\text { Arguments }\end{array}$ & Operations & Results \\
\hline Modis.exe & MODIS(.hdf) & $\begin{array}{l}\text { Data path, Scope } \\
\text { control info, Cloud } \\
\text { threshold }\end{array}$ & $\begin{array}{l}\text { Geometric } \\
\text { calibration, } \\
\text { Radiometric } \\
\text { calibration, } \\
\text { Atmospheric } \\
\text { correction }\end{array}$ & $\begin{array}{l}\text { Cloud image, NDVI } \\
\text { image, False color } \\
\text { image }\end{array}$ \\
\hline hj.exe & $\begin{array}{l}\mathrm{HJ}-1 \\
\mathrm{CCD}(. \mathrm{XML}+. \mathrm{tif})\end{array}$ & $\begin{array}{l}\text { Data path, Cloud } \\
\text { threshold }\end{array}$ & $\begin{array}{l}\text { Radiometric } \\
\text { calibration, } \\
\text { Atmospheric } \\
\text { correction }\end{array}$ & $\begin{array}{l}\text { Cloud image, NDVI } \\
\text { image, False color } \\
\text { image }\end{array}$ \\
\hline cosmo.exe & COSMO(.h5) & Data path & $\begin{array}{l}\text { Radiometric } \\
\text { calibration }\end{array}$ & $\begin{array}{l}\text { Calibrated } \\
\text { image }\end{array}$ \\
\hline radarsat.exe & RADARSAT(.tif) & Data path & $\begin{array}{l}\text { Geometric } \\
\text { calibration, } \\
\text { Radiometric } \\
\text { calibration }\end{array}$ & $\begin{array}{l}\text { Calibrated } \\
\text { image }\end{array}$ \\
\hline terra.exe & Terra SAR-X(.tif) & Data path & $\begin{array}{l}\text { Radiometric } \\
\text { calibration }\end{array}$ & $\begin{array}{l}\text { Calibrated SAR } \\
\text { image }\end{array}$ \\
\hline greentide.exe & All above & $\begin{array}{l}\text { Image data path, } \\
\text { Roi (.shp) path }\end{array}$ & $\begin{array}{l}\text { Green tide coverage } \\
\text { points extraction }\end{array}$ & $\begin{array}{l}\text { Green tide coverage } \\
\text { information } \\
\text { value image) }\end{array}$ \\
\hline
\end{tabular}

Table 1 Statistics for Green Tide Remote Sensing Image Processing Modules

The calling process of the modules above in the system is shown in Figure 2.First of all, five different types of optical and microwave remote sensing data are set as source data and several image pre-processing operations, such as radiometric calibration, geometric correction, cloud detection, atmospheric correction, false color synthesis, are carried out according to respective 
requirements of different types of data through invoking corresponding image pre-processing modules(EXE) to obtain pre-processing results such as NDVI images, Calibrated SAR images, cloud images, false color images. Then, the NDVI images or calibrated SAR images achieved in the last step are set as the source data. The green tide coverage information extraction module, where a series of operations including reading interpreted image file, cutting ROI based on cloud images and land files, processing the source data with the ROI mask, determining threshold according to SAR or NDVI

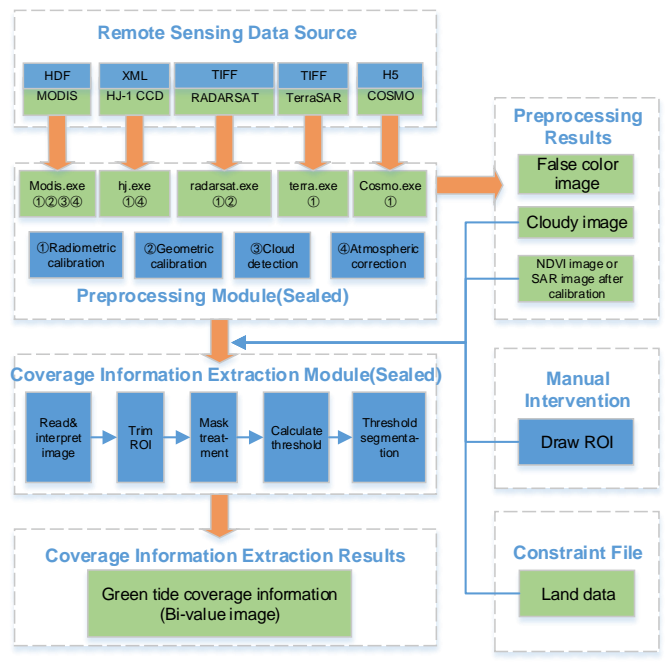

Figure. 2 The flow chart of multi-source remote sensing data preprocessing and green tide coverage information extraction

histogram, image segmentation using threshold, are performed, is invoked. Finally, the extraction result of green tide remote sensing information in ROI, that is, bi-value images containing green tide coverage information, is obtained.

(2) Unified data format:

All process data in this paper are stored in a uniform data storage format and uniform naming rules. All raster data is stored in GeoTIFF format and all vector data is stored in Shapefile format. File naming rules are unified as 'type of original data_12 bit time string_type of result_resolution_processing time', for example, 'modis_201510151605_ndvi_250_1612.tif'.

(3) Unified image property standard:

The Nodata value in the image preprocessing result is set to 0 because Nodata values of images are uniformly assigned to special numbers, like -9999, in ENVI, while invalid values of images default to positive infinity, negative infinity or 0 in ArcGIS

(4) Unified management methods:

Due to the large quantities of raster and vector data generated in image processing and in the process of green tide distribution information extraction, therefore one separate folder is created as workspace where relative data are stored every day. Unnecessary data shall not be preserved.

\section{GREEN TIDE DISTRIBUTION CONTOUR EXTRACTION ALGORITHM}

The bi-value images extracted from green tide coverage information, and the vector point data transformed from bi-value images are discrete information of green tide, as is shown in Fig.3, where there is no division and distribution information of green tide blocks for operational green tide remote sensing monitoring system.

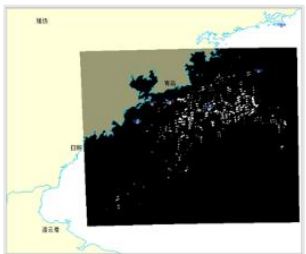

(a)Bi-value image

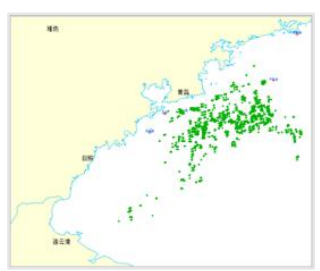

(b)Vector Data
Figure 3. The Green Tide Coverage Information

Green Tide Distribution Contour Extraction here refers to the process of extracting the boundary lines of all discrete points containing green tide coverage information. The traditional processing method connects the boundary points directly to extract the scattered contours, as shown in Fig.4. However, it is difficult to determine the appropriate boundary points during operation, which is not conducive to batch processing of the program, and the contour line obtained is abruptly connected. Therefore, an automatic distribution contour extraction algorithm for green tides is designed in this study.

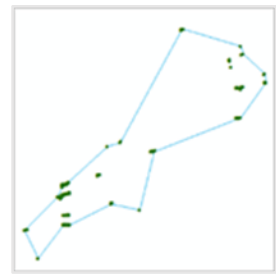

Figure. 4 The Effect Diagram of Direct Extraction Method

\subsection{Algorithm Design}

According to Code of Practice for Marine Monitoring Technology promulgated by the State Oceanic Administration, the division method of green tide blocks is defined as follows: 
Any two green tide areas on the map are considered to be two separate green tide blocks if the minimum distance between the outermost ends of them is greater than or equal to a certain distance $(10 \mathrm{~mm}$ on the screen, i.e., fusion distance, FDis), otherwise to be one single block, as shown in Fig. 5. For a single green tide block, the inner range of the envelope created by extrapolating a distance $(0.5 \mathrm{~mm}$ on the screen, i.e., extrapolation distance, EDis) from the edge of green tide coverage is defined as the spatial distribution of the block. Because the green tide discrete points extracted from the remote sensing images characterized by massive data and irregular distribution, an efficient and accurate algorithm for dividing huge amount of green tide coverage discrete points into blocks referring to fusion distance and for extracting the contour of the blocks.

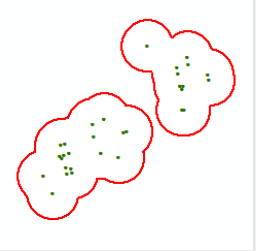

Figure. 5 The Sketch Map of Block Division

Buffer analysis is a commonly used neighborhood analysis method, which is simple and easy to understand. No more detail is discussed here.

The application of mathematical morphology on images can simplify image data, maintain their basic shape features, and remove irrelevant structures. Morphological closed operations are often used to fill small holes in an image and smooth the boundaries of images. After closed operation, the overall shape and area of the image do not change significantly. The expression of morphology closed operation is as follows:

$$
f \cdot b=(f \oplus b) \ominus b
$$

In the expression, $f$ refers to the object and $b$ refers to the structural element. $f$ expands according to $b$ and subsequently the result of expansion corrodes by $b$.

The two methods are applied directly to the green tide discrete data to extract the distribution information, and the results are obtained respectively as shown in Fig.6(a) and Fig.6(b). In Fig.6(a), buffer analysis is applied and buffer areas of every green tide discrete point are created with the fusion distance as buffer distance. The overlapping buffer areas are fused to obtain the area surrounded by the red border. After that, buffer area for the red border area is made with the opposite number of fusion distance as buffer distance, in order to basic green tide distribution contour, as the blue polyline shown in Fig.6(a). While in Fig.6(b), mathematical morphological closed operation is applied on green tide bi-value image. Firstly, the green tide coverage area is dilated to the blue area, which represents the block partition of green tide; then it is eroded to red area, which represents the basic distribution of green tide.
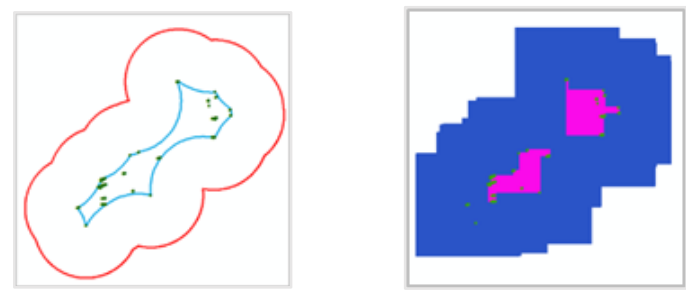

$\begin{array}{ll}\text { (a) Buffer Scheme } & \text { (b) Closed Operation Scheme }\end{array}$

Figure. 6 The Effect Diagram of Buffer Scheme and Closed

Operation Scheme

The two methods both have their own advantages and disadvantages here. The green tide distribution obtained by the buffer analysis method is smooth and accurate, however, the detailed description of this method leads to excessive computational complexity. Mathematical morphology closed operation method is able to process massive green tide data, but the obtained contour is abrupt and does not accurately describe the boundaries of the green tide distribution.

An attempt is made in this study to explore a green tide distribution contour extraction method that not only combines the advantages of both buffer analysis method and closed operation method, but also avoid their disadvantages, in order to ensure it applicable to actual business applications.

Here, the Euclidean distance analysis is used as the dilation process of the closed operation, and the inner buffer is used as the corrosion process of it. As is shown in Fig.7: the green tide coverage points are used as source data (as the green points shown in the figure). Firstly, Euclidean distance analysis is performed as "expansion", where the key parameter, "the maximum distance" (the yellow radiations in Fig.7), is specified as the fusion distance, to obtain a gray-scale image like a fused overlay buffer fusing nearest neighbor related green tide coverage information in the same block. After that, condition analysis is performed on the gray-scale image to obtain the bivalue image and then it is transformed into a vector file through 
raster-vector conversion and the block information (as the red border area shown in Fig.7) is extracted. Finally, the inward buffer (buffer distance is the negative value of fusion distance) is made for the resulting block to operate "corrosion" (as the purple radiations in Fig.7) and to obtain the basic distribution of green tide (as the blue border area in Fig.7). And the buffer for the basic distribution is made with the extrapolation distance as buffer distance to obtain the basic distribution after extrapolation.

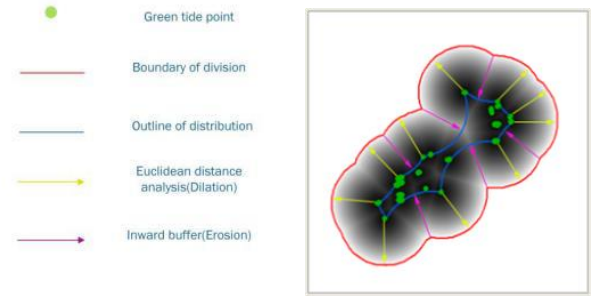

Figure.7 The Sketch Map Green Tide Distribution Contour Extraction Algorithm

\subsection{Algorithm Implementation}

The whole process of green tide distribution contour extraction algorithm is shown in figure 8 . In order to avoid isolated green tide point information missing in the process of inward buffering due to the absolute value of buffer distance equal to fusion distance. Firstly, the green tide blocks are divided, and then the basic distribution contours are obtained according to the types of the division results.

(1) Data input and parameter setting

The bi-value images or discrete vector points obtained from green tide coverage information extraction are read, and the constraint files like land files, cloud files are imported. The key parameters involved in the algorithm are set.

(2) Green tide block partition

Distance analysis: the cluster analysis tool based on Euclidean distance analysis is used to measure the straight-line distance between each pixel center and its nearest green tide pixel center. The green tide vector point data or bi-value images were entered. The output grid pixel size, cell_size, is set and the maximum distance is specified as the fusion distance. The result of the operation is the gray image whose gray level represents the distance value where the closer the distance from the green tide point is, the bigger the gray value is, the darker $t$ 、 e color is, and vice versa; Condition analysis: in the gray-scale image obtained in distance analysis, the areas represented by gray scale are the divided blocks, whose gray values of pixels are all greater than or equal to 0 , while the rest of the areas that are transparent, are not in the blocks, where the gray values of pixels are all NoData
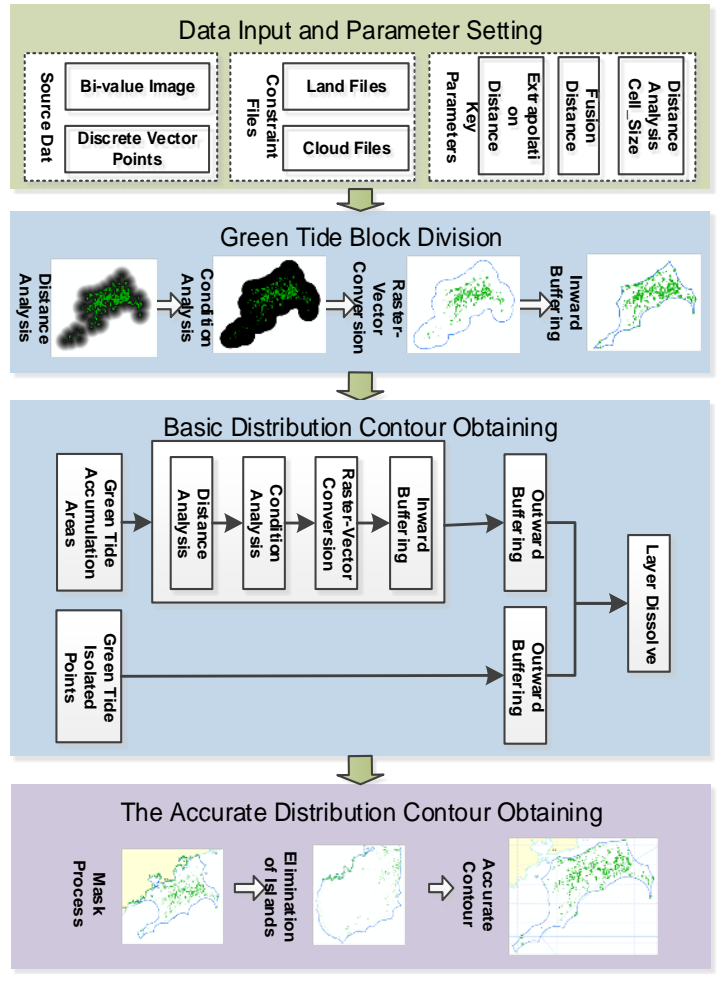

Figure. 8 Flow Chart of Green Tide Distribution Contour

Extraction

$(-3.4028235 \mathrm{e}+038)$. Therefore, condition analysis tool is used to make condition judgement on the image pixel by pixel to assign the gray values of pixels to 1 whose gray value are greater or equal to 0 originally. And the rest of the gray values remain NoData as default. The bi-value image that represents green tide blocks division is obtained;Raster-vector conversion: the rastervector tool is used to covert the bi-value image representing green tide block partition into vector polygon, so as to operate buffer analysis;Inward buffering: After distance analysis, green tide discrete points have been expanding a fusion distance (FDis) At that time, make an inward buffer with 1/2cell_size-FDis, whose absolute value is a little smaller than fusion distance, as buffer distance to make sure that all the green tide information be covered in blocks and to avoid the problem that isolated green tide information cannot be extracted. The result is used to distinguish between green tide accumulation areas and isolated points.

(3) Basic distribution obtaining

For green tide accumulation areas, the operation in the previous step is repeated on the discrete points in each block. However, the difference is that after the raster-vector conversion, an inward buffer is directly made with the opposite number of fusion distance as buffer distance, then an outward buffer is made with 
extrapolation distance as buffer distance and fuse all the blocks by the way of merging polygon layers to obtain the basic distribution of green tide accumulation areas. While for isolated green tide information, only an outward buffer with the extrapolation distance as buffer distance is needed to obtain the basic distribution here.

(4) Obtaining of accurate distribution contour of green tide In order to meet the spatial constraints and business needs in practical application, land files and cloud files are used as mask to operate mask processing on the obtained contour of basic green tide distribution, and the features such as "islands" and "rings" are eliminated by the way of dissolving the polygons. Finally, accurate extraction of green tide distribution contour is accomplished and the vector files of green tide distribution that meet business need are obtained.

\subsection{Algorithm Verification}

As is shown in Fig.6, (a) is 250m resolution Modis image; (b) is the green tide distribution information obtained through green tide coverage information extraction from the Modis image and distribution information extraction from its result; (c) presents the effect that green tide coverage information and distribution information are overlaid on the original remote sensing data.

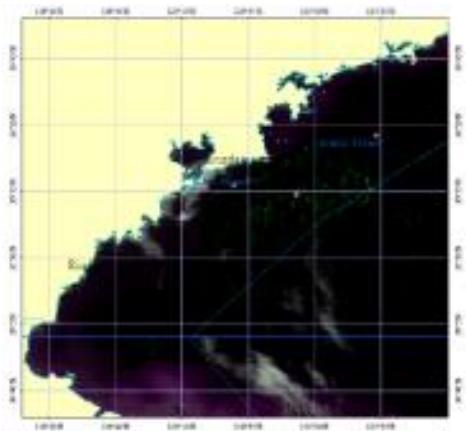

(a)Remote Sensing Image

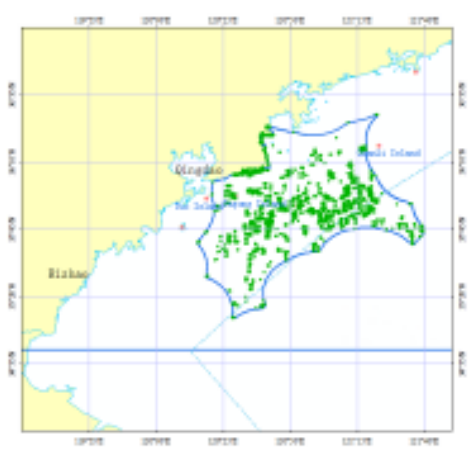

(b)Distribution Information Map

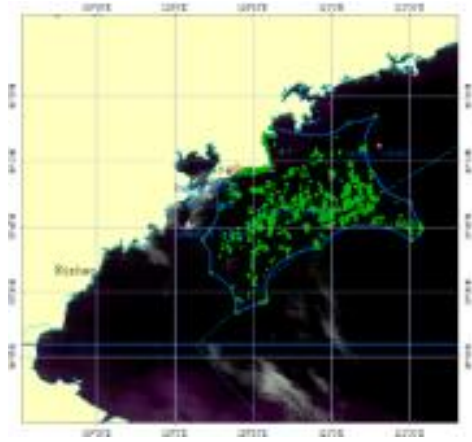

(c) Image Interpretation Map

Figure. 9 The Verification of Green Tide Distribution Contour Extraction Algorithm

It can be seen from overlay that, the obtained green tide distribution contour exactly and completely surrounds all the green tide discrete points, and the contour is smooth and accurate, which absolutely meets the need of operational green tide monitoring.

\section{CONCLUSION}

In this study, the integration of remote sensing and GIS environment is realized by using the sealed modules of remote sensing images processing and green tide coverage information extraction to deal with 5 different types of optical RS data and microwave RS data in the system. And a new efficient green tide distribution contour extraction algorithm which combines Euclidean distance analysis and closed operation in mathematical morphology has been explored. The green tide remote sensing monitoring system not only achieves integration of remote sensing image processing and spatial analysis and visualization of GIS, but also raises the efficiency and accuracy of green tide spatial distribution extraction, which is in favor of helping marine sectors realize one-click data processing and provide quick decision-making support. At present, the system has been widely used in business sectors. In the following study, the green tide coverage information extraction method and green tide distribution contour extraction algorithm will be further optimized, and relative functions of the system will be extended. 


\section{REFERENCE}

Dekker, A.G., Peters, S.W.M,. 1993. The use of the Thematic Mapper for the analysis of entropic lakes, a case study in the Netherlands. International Journal of Remote sensing. 14:799822.

Hu, C., He, M. X., 2008. Origin and offshore extent of floating algae in Olympic sailing area. Eos,American Geophysical Union Transactions. 89(33): 302-303.

Jeffrey, D.W., 1993. Sources of nitrogen for muisance macroalgal growths in Dublin Bay, Republic of Ireland. Phycologist. 34:30.

Liu, D., Keesing, J. K. , Xing, Q., et al. 2009. World's largest macroalgal bloom caused by expansion of seaweed aquaculture in China. Marine Pollution Bulletin. 58: 888-895.

Ruifu, Wang., 2011. Design and Implementation of Coastal Zone Remote Sensing InformationExtraction Platform. Journal of Software. 6(7), 1188-1193.

Ruifu, Wang., Nannan, Liu., Maojing, Xu., Xiangchao, Kong., 2014. Research on the Open Source GIS Development Oriented to Marine Oil Spill Application. Journal of Software. 9(1), 116120.

Ruifu, Wang., 2011. Data Interoperability Analysis of MIF in ArcGIS Environment. Geoinformatics. 1-4.

Ruifu, Wang., Maojing, Xu., Yi, Ding., Nannan, Liu., Xiangchao, Kong., 2014. The Development of Marine Oil Spill Operational System Based on GIS. Journal of Software. VOL. 9, NO. 8, 2050-2055.

Ruifu, Wang., 2010. Map Decoration Implemented Based on Element under ArcGIS Environment. GeoInformatics. 1-5.

Raffaelli, D.G., Raven, J.A., Poole., L.J., 1998. Ecological impact of green macroalgal blooms. Oceanography and Marine Biology:an Annual Review. 199836:97-125.
Wang, M., Shi, W., 2008. Satellite observed algae blooms in China's Lake Taihu. Eos Trans. AGU. 89: 201-202.

Xu, Jiangling., Huang, Juan., Gao, Song., Cao, Yajing., 2014. Assimilation of High Frequency Radar Data into a Shelf Sea Circulation Model. Journal of Ocean University of China. 13 (4): $572-578$

Xiangchao, Kong., Ruifu, Wang., 2013. Research on Mapping Technology of GIS Based on Business-oriented Marine Remote Sensing Monitoring. GeoInformatics. 1-3.

ZHAO, Jian., CHEN, Xueen., XU, Jiangling., HU, Wei., CHEN, Jinrui., Pohlmann Thomas., 2013. Assimilation of surface currents into a regional model over Qingdao coastal waters of China. Acta Oceanologica Sinica. 7,21-28. 